\title{
Setting Apart the Affected: The Use of Behavioral Criteria in Animal Models of Post Traumatic Stress Disorder
}

\author{
Hagit Cohen*,', Joseph Zohar', Michael A Matar', Kaplan Zeev', Uri Loewenthal' and Gal Richter-Levin ${ }^{3}$ \\ 'Anxiety and Stress Research Unit, Ministry of Health Mental Health Center, Faculty of Health Sciences, Ben-Gurion University of the Negev, \\ Beer- Sheva, Israel; ${ }^{2}$ The Chaim Sheba Medical Center, Sackler Medical School, Tel-Aviv University, Israel; ${ }^{3}$ Department of Psychology, University \\ of Haifa, Israel
}

\begin{abstract}
Post-traumatic stress disorder (PTSD) affects about 20-30\% of exposed individuals. Clinical studies of PTSD generally employ stringent criteria for inclusion in study populations, and yet in animal studies the data collection and analysis are generally expressed as a function of exposed vs nonexposed populations, regardless of individual variation in response. Prior data support an approach to animal models analogous to inclusion criteria in clinical studies. This series of studies sought to assess prevalence rates of maladaptive vs adaptive responses determined according to a more stringent approach to the concept of inclusion/exclusion criteria (cutoff behavioral criteria-CBC), consisting of two successive behavioral tests (elevated plus maze and acoustic startle response tests). The rats were exposed to stressors in two different paradigms; exposure to a predator and underwater trauma. The prevalence rates of maladaptive responses to stress in these two distinct models dropped over time from $90 \%$ in the acute phase to $25 \%$ enduring/maladaptive response at 7 days, to remain constant over 30 days. As setting the affected individuals apart from the unaffected approximates clinical studies, it might also help to clarify some of the pending issues in PTSD research.

Neuropsychopharmacology (2004) 29, 1962-1970, advance online publication, I4 July 2004; doi: I 0. I 038/sj.npp. 1300523
\end{abstract}

Keywords: post-traumatic stress disorders; animal models; anxiety; predator stress; acoustic startle response; elevated plus maze; maladapted; well adapted

\section{INTRODUCTION}

Post-Traumatic Stress Disorder (PTSD) is an incapacitating chronic clinical syndrome ensuing from exposure to traumatic events involving a perceived threat to life or physical integrity (American Psychiatric Association, 1994). Its symptoms are divided into three clusters and must be present over a period of at least 1 month: (1) intrusive reexperiencing of the traumatic event, (2) persistent avoidance of stimuli associated with the trauma and emotional numbing, and (3) persistent symptoms of increased physiological arousal (American Psychiatric Association, 1994). Similar symptoms that significantly compromise the individual's functioning at an earlier time qualify for the diagnosis of Acute Stress Disorder.

Retrospective and prospective epidemiological studies of PTSD indicate that only a proportion of the population

* Correspondence: Dr H Cohen, Anxiety and Stress Research Unit, Ministry of Health Mental Health Center, Faculty of Health Sciences, Ben-Gurion University of the Negev, Beer-Sheva 84170, Israel, Tel: + 9728 6401743, Fax: 9728 6401742,

E-mail: hagitc@bgumail.bgu.ac.il.

Received 12 January 2004; revised I June 2004; accepted 4 June 2004 Online publication: 16 June 2004 at http://www.acnp.org/citations/ Npp06 1604040 I4/default.pdf exposed to any specific event will develop the full-blown chronic syndrome. In the US, studies report that the rate of lifetime exposure to at least one serious traumatic event (excluding grief and mourning) is quite high; a conservative estimate reported $61 \%$ among men and $51 \%$ in women (Kessler et al, 1995). Other studies have found similar rates (Breslau et al, 1991; Breslau et al, 1998; Helzer et al, 1987; Kessler et al, 1995; Perkonigg et al, 2000; Resnick et al, 1993; Shore et al, 1989). The lifetime prevalence of PTSD in the general population reaches about $7 \%$ overall (Fairbank et al, 2000 ), suggesting that about $20-30 \%$ of individuals exposed to severe stressors will develop PTSD (Breslau et al, 1991). This figure varies depending on the type of trauma studied, where male rape victims suffer very high rates and populations exposed to natural disasters significantly less (Shalev, 2000).

The marked difference between the proportion of the general population exposed to potentially traumatizing events and the proportion who develop the full-blown clinical disorder suggests possible qualitative differences in terms of factors related to vulnerability and/or resilience between those individuals who were exposed and developed the full-blown syndrome $v s$ those who did not.

In order for the animal model of a human condition to be useful in advancing clinical understanding, it must be as 
valid as possible an approximation of the human disorder it is modeling. The rationale behind the use of animal models for human conditions is to enable experimentation in ways and with sample sizes, which are many times impossible in humans, for ethical/moral or practical/technical reasons. Extrapolation from such models to the human conditions must be carried out with care, always keeping in mind that models are no more than partial approximations, and that there is an inherent risk of overhumanizing animal behaviors.

Over the past decades, a variety of animal models have been put forward as being valid for the study of stress and anxiety, and of their chronic or long-term effects, such as inescapable electric (foot) shock (Pynoos et al, 1996; Servatius et al, 1995), social confrontations (Stam et al, 2000), underwater trauma (Richter-Levin, 1998; Wang et al, 2000), and exposure to a predator (Adamec, 1997; Adamec $\mathrm{R}$ et al, 1998; Adamec RE et al, 1999; Adamec and Shallow 1993; Adamec RE et al, 1997; Cohen et al, 2000; Cohen et al, 1996; Cohen et al, 2003; Cohen et al, 1999). Various behavioral tests and biophysiologic measures have been performed ensuing the exposure.

Irrespective of the design of the model, the data from these studies have routinely been reported and interpreted uniformly for the entire exposed population, regarding it as a homogeneous whole. However, in practice, there is considerable variability in the degree of behavioral disruption or response to the stressor and animals tend to demonstrate considerable individual differences, certainly in the degree to which their behavior is disrupted.

Human responses to traumatic stressors clearly vary greatly, in extent and in character. This has formed the basis for contemporary definitions of stress-related disorders according to diagnostic tools such as the DSM or ICD, and for inclusion and exclusion criteria applied in many clinical trails.

In a previous study, the authors examined the effect on the analysis of the data of segregating the study population of exposed animals into those individuals that were clearly behaviorally affected (maladapted, MA) $v s$ those individuals clearly unaffected in spite of having been exposed to the stressor (well-adapted, WA), according to their observed behaviors on the elevated plus maze (EPM) paradigm.

The range of the degree of behavioral response was fairly broad, although there was a clearcut cluster of individuals grouped at the very extreme end of the range, whose behavior was most significantly disturbed, and which was significantly different from that of the rest of the population. Accordingly, phenomenologically determined cutoff behavioral criteria (CBC) were determined for the upper (highly disturbed) and lower (virtually unaffected, ie equivalent to control animals) extremes of behavioral change (Cohen et al, 2003).

Applying this mode of examining the data in tandem with the standard approach revealed that only about $25 \%$ of the rats developed significant behavioral disruptions (MA) as the result of exposure to the stressor, as compared to $1.3 \%$ in unexposed controls. The prevalence of unaffected (WA) rats was only $24.7 \%$ in the exposed groups, as compared to $80 \%$ in the control group. Moreover, in comparison to controls and WA exposed rats, MA rats exhibited distinct physiologic parameters - namely, significantly higher plasma corticosterone and ACTH concentrations, increased sympathetic activity, diminished vagal tone, and increased sympathovagal balance.

The authors therefore suggested that application of $\mathrm{CBC}$ is not only feasible but might also allow more focused study of the pathological effects ensuing from exposure to trauma, by excluding data from animals that are minimally affected or unaffected by the paradigm or, conversely, focusing on these data intentionally.

The aim of the present study was to further examine the feasibility and validity of applying a more stringent version of the $\mathrm{CBC}$ concept in animal studies, using two consecutive behavioral paradigms, rather than one. The authors also examined the consequences of applying the CBC method of assessment in animals exposed to different stressparadigms. In each of these two models, the response was to be evaluated in terms of magnitude of response among the exposed population as a function of time elapsed.

The requirement for maladaptive responses to two paradigms was intended to further refine the distinction between the MA and WA groups in order to more closely approximate this animal model to the current diagnosing practice for PTSD in human subjects.

\section{MATERIALS AND METHODS}

\section{Model 1: Predator Exposure (Cat)}

Blanchard et al (1990, 1990b, 1998, 1993), Griebel et al (1995a, b), Rodgers et al (1990), and Shepherd et al (1992) were the first to examine the responses of wild and domestic rats to feline predators. Brief escapable exposure to a cat or cat odor increased defensive behaviors in a visible burrow system for many hours after removal of the threat. Predatory stimuli are ecologically relevant for an animal's survival and, consequently, induce responses quite similar to those shown in natural contexts. In our model, we exposed rats to a cat for $10 \mathrm{~min}$ as previously described by Adamec et al $(1999,1997)$, Adamec and Shallow (1993), and Cohen et al $(2000,1996,1999)$.

\section{Experimental procedures.}

Animals: A total of 240 adult male Sprague-Dawley rats weighing $150-200 \mathrm{~g}$ were employed. Animals were habituated to the housing conditions for at least 10 days. During that time, rats were handled once daily (consisting of picking the rats up with a gloved hand). The animals were housed four per cage in a vivarium with stable temperature and reversed 12-h light/dark cycle, with ad libitum food and water. All testing was performed during the dark phase using a dim light.

Six consecutive studies were performed, using identical behavioral paradigms under different days after each exposure. Behavioral reactions were tested $1,3,5,7,14$, and 30 days after each exposure.

Rats were tested in two behavioral models - the EPM and the acoustic startle response (ASR) paradigm.

Exposure to cat-litter urine for $10 \mathrm{~min}$ : The stress paradigm consisted of placing the test animals on wellsoiled cat-litter for $10 \mathrm{~min}$ (in use by the cat for 2 days, 
sifted for stools). The control animals were exposed to fresh, unused litter for the same amount of time.

\section{Model II: The Underwater Trauma}

Underwater trauma (Richter-Levin, 1998) is a tangible lifethreatening situation which is designed to model sudden, brief traumatizing experiences. It has a number of appealing qualities as a model for acute and chronic reactions to stress. Firstly, it is intuitively a much more naturally occurring situation than electrical foot or tail shocks for example. It also represents a deep-rooted primal terror of smothering and suffocation. Secondly, the Morris water maze memory task comes to represent a model of in-context retraumatization and thus become a testing ground of special significance for the study animals. The importance of restressing animals in context, rather than out of context, was clearly demonstrated in a previous report by RichterLevin (1998), in which significant effects on performance were demonstrated when the trauma was in context, as opposed to out-of-context.

\section{Experimental procedures.}

Animals: A total of 72 adult male Sprague-Dawley rats weighing 150-200 g were employed. Animals were habituated to the housing conditions for at least 10 days. During that time, rats were handled once daily (consisting of picking the rats up with a gloved hand). The animals were housed four per cage in a vivarium with stable temperature and reversed 12-h light/dark cycle, with ad libitum food and water. All testing was performed during the dark phase using a dim light.

Rats in the trauma group were given $1 \mathrm{~min}$ to swim in a circular pool ( $1.8 \mathrm{~m}$ in diameter and $0.6 \mathrm{~m}$ high), containing water at $26 \pm 1{ }^{\circ} \mathrm{C}$ and then held under water for $30 \mathrm{~s}$ using a metal net. Control rats were given $1 \mathrm{~min}$ to swim and then put in a resting cage.

Experiments for the underwater trauma model: Three consecutive studies were performed, using identical behavioral paradigms (the EPM and the ASR paradigm). Behavioral reactions were tested 1,5 , and 30 days after each exposure. A total of 24 rats were used in each experiment: 12 for the exposed group and 12 for the control group.

\section{Behavioral paradigms.}

The EPM: The maze employed is a four-armed black opaque perspex platform, elevated $50 \mathrm{~cm}$ above the ground, as described by File (1993). Two opposite arms are enclosed by $40 \mathrm{~cm}$ high perspex walls on both sides and on the outer edges of the platform, that is, closed, while the two remaining opposite arms are open, surrounded by a $1 \mathrm{~cm}$ high perspex lip, which serves as a tactile guide to animals in the open areas. The apparatus is illuminated by dim red lighting, arranged in such a manner as to provide 40-60 lux in both open and closed arms. Individual rats were placed in the central platform, faced towards different arms on different days in randomized order. The maze was cleaned with a 5\% ethanol/water solution and dried thoroughly between test sessions.
Procedure: Each 5-minute session was recorded using an overhead video camera connected to a monitor/recorder in an adjacent observation room. Observer's blind to group allocations, rated behaviors by reviewing taped sessions.

Five measures of behavior were assessed: (1) time spent in the open arms; (2) time spent in the closed arms; (3) the number of entries into open arms; (4) the number of entries into closed arms; (5) total entries into all arms. Animals were scored as being in an open or closed arm only when all four paws passed over the open/closed dividing line. Exploration activity was calculated as the number of entries into any arm of the maze (total arm entries).

ASR: The animals were tested in pairs with startle chambers counterbalanced across the different experimental groups. Startle response was measured using two ventilated startle chambers (SR-LAB system, San Diego Instruments, San Diego, CA). Each chamber consisted of a Plexiglas cylinder resting on a platform inside a soundattenuated, ventilated chamber. A high-frequency loudspeaker inside the chamber produced both a continuous broadband background noise of $68 \mathrm{~dB}$ and the various acoustic stimuli. Movement inside the tube was detected by a piezoelectric accelerometer below the frame. The amplitude of the whole body startle to an acoustic pulse was defined as the average of 100 one-millisecond accelerometer readings collected from pulse onset. These signals were then digitized and stored by a computer. Sound levels within each test chamber were measured routinely using a sound level meter (Radio Shack) to ensure consistent presentation. The SR-LAB calibration unit was used routinely to ensure consistent stabilimeter sensitivity between test chambers and over time. The animals were placed inside the tube, the startle session started with a 5-min acclimatization period, with a background noise level of $68 \mathrm{~dB}(\mathrm{~A})$, which was maintained throughout the session.

\section{Behavioral Assessments for Determination of CBC}

Study design: CBC. The studies were designed in two steps:

Step I: Prior to attempting to distinguish the differentially affected subgroups, we routinely perform a preliminary assessment of the overall response of the exposed population intended to ascertain the accuracy of our zerohypothesis, that is to demonstrate that exposure to the stressor did in fact have significant overall behavioral effects on the exposed rats as a group compared to controls, in each of our studies. The data are also routinely ascertained to demonstrate a range of varying degrees of behavioral changes.

Behavioral changes, such as extremely compromised exploratory behavior on the plus maze and markedly increased startle reaction that does not undergo any adaptation, reflect anxiety-like behaviors, that is, fearfulness and hypervigilance. In keeping with the work of Blanchard et al (1990, 1998, 1993), Shalev (2000), Adamec (1997), Adamec R et al (1998), Adamec RE et al (1999, 1997), and our own previous studies (Cohen et al (2000, 1996, 2003, 1999), the observed behaviours at this time point are considered to reflect relatively long-term and persistent 
changes. Since it has as yet not been possible to design an animal model for the intrusive cluster of symptoms, changes such as these, which persist over the space of a week or more, are considered a fair representation of PTSDlike symptoms in terms of animal models.

Step II: the CBC applied to exposed rats: Having established that the stressor had an effect on the rats and that not all animals responded to it in the same manner, we focus only on animals that demonstrate extremes of behavioral change on the one hand or virtually no change on the other.

In order to maximize the accuracy of which animals to define as affected, and so to minimize the chance of including false positives, we define the behavioral cutoff criteria to represent the most extreme degree of behavioral disturbance in each of two consecutive behavioral paradigms. In order to be defined as affected, the individual animal has to have conformed to both sets of criteria, consecutively. Conversely, in order to be considered to have responded hardly at all, animals must conform to equally extreme criteria for near normal behaviors. The validity of the criteria are reaffirmed in each study by ascertaining that the vast majority of unexposed control animals conform to the latter and none, or almost none, to the former.

The CBC determined as above, were as follows:

\section{Maladapted}

(1) 5 min spent in closed arms and zero (0) entries into the open arms

(2) Mean amplitude of the startle response (at $110 \mathrm{Db})>$ 1200 units and no habituation over time.

Well-Adapted

(1) $0-1$ min spent in closed arms and $\geqslant 8$ open-arm entries.

(2) Mean amplitude of the startle response (at $110 \mathrm{Db}$ ) $<1000$ units and normal habituation of the ASR.

\section{Statistical Analyses}

The differences in the general shapes of the distributions of the EPM parameters in the exposed group and the control group were analyzed using Kolmogorov-Smirnov test. Owing to the skewed variance in the EPM measure, the significance of changes and differences were assessed by the nonparametric Mann-Whitney $U$-test.

For the ASR results, the statistical analyses were performed using one-way analysis of variance (ANOVA).

To gain additional information about the relationship between the behavioral parameters data, the Pearson correlations were computed.

The prevalence of the affected rats was tested using $\chi^{2}$ tests.

\section{RESULTS}

\section{Model 1: Predator Exposure}

The effect of a single exposure to a predator on anxietylike behaviors. The distributions of the exposed groups and the control group (time spent in the closed arms) were compared by the Kolmogorov-Smirnov statistical test. The results showed that there are differences in the general shapes of the distribution curves in the two populations $(p<0.01)$. The comparison between exposed rats $v s$ controls in each experiment was thus made using the MannWhitney $U$-test. The behavioral other parameters (in the plus maze) were evaluated using the $t$-test (normal distribution).

As shown in Table 1 a single 10-min exposure to a cat caused significant avoidance of open spaces as compared to control (nonexposed) animals as measured 1-30 days after the exposures. Exposed rats spent significantly more time exploring the closed arms of the EPM $(p<0.0001)$ and entered more frequently into the closed arms $(p<0.0001)$ than the control rats. However, total entries into the open and closed arms (total arms entries) of the plus maze were not reduced in all the experiments.

The comparison between exposed rats between the experiments (numbers of days after exposure) was made using Kruskal-Wallis test. In the parameters, time spent in the closed arms, the Kruskal-Wallis test revealed significant difference between experiments $(\mathrm{H}(5,120)=$ 25.5, $p<0.0001)$.

Using the Mann-Whitney $U$-test, 1 day after exposure, the exposed rats spent significantly more time exploring the closed arms of the EPM than 5 days $(Z=2.9, p<0.004)$, 7 days $(Z=3.2, p<0.002)$, 14 days $(Z=3.35, p<0.0008)$, or 30 days $(Z=3.3, p<0.0001)$ after exposure.

The effect of a single exposure to a predator on ASR. As shown in Table 1, a single 10-minute exposure to a cat significantly increased the mean startle response as compared to control (nonexposed) as measured 1-30 days after the initial exposure $(p<0.01)$.

The comparison between exposed rats between the experiments was made using ANOVA and revealed a significant difference between experiments $(F=(5,114)=$ 4.6, $p<0.0005)$. Post hoc Scheff test reveled that exposed rats day $1 \neq$ exposed rats at day $30(p=0.008)$.

Correlation of the time spent in the closed arms parameter and ASR. In the exposed group, there was a significant correlation between time spent in the closed arms and startle response $(r=0.57, \mathrm{~F}(1,118)=56.5, p<0.00001)$.

In the control group, there were no significant correlations between time spent in the closed arms and startle response.

\section{Reanalysis of data applying the $C B C$.}

The prevalence of maladapted rats: Figure 1a shows a steady drop in prevalence of maladapted rats from $90 \%$ on day 1 after cat exposure to $24 \%$ by day 7 . As of day 7 , this rate remains constant until day 30 . Our results show a progressive, time-dependent decline in the prevalence of MA rats during the first few weeks that follow a trauma.

As shown in Figure 1b, most exposed animals demonstrated at least same degree of response in either behavior or startle response after exposure and at first minimal behavioral response (WA) animals were almost impossible to find among the exposed rats using both consecutive 
Table I Effect of Single Exposure to Predator on Rat Anxiety-Like Behavior and Acoustic Startle Response

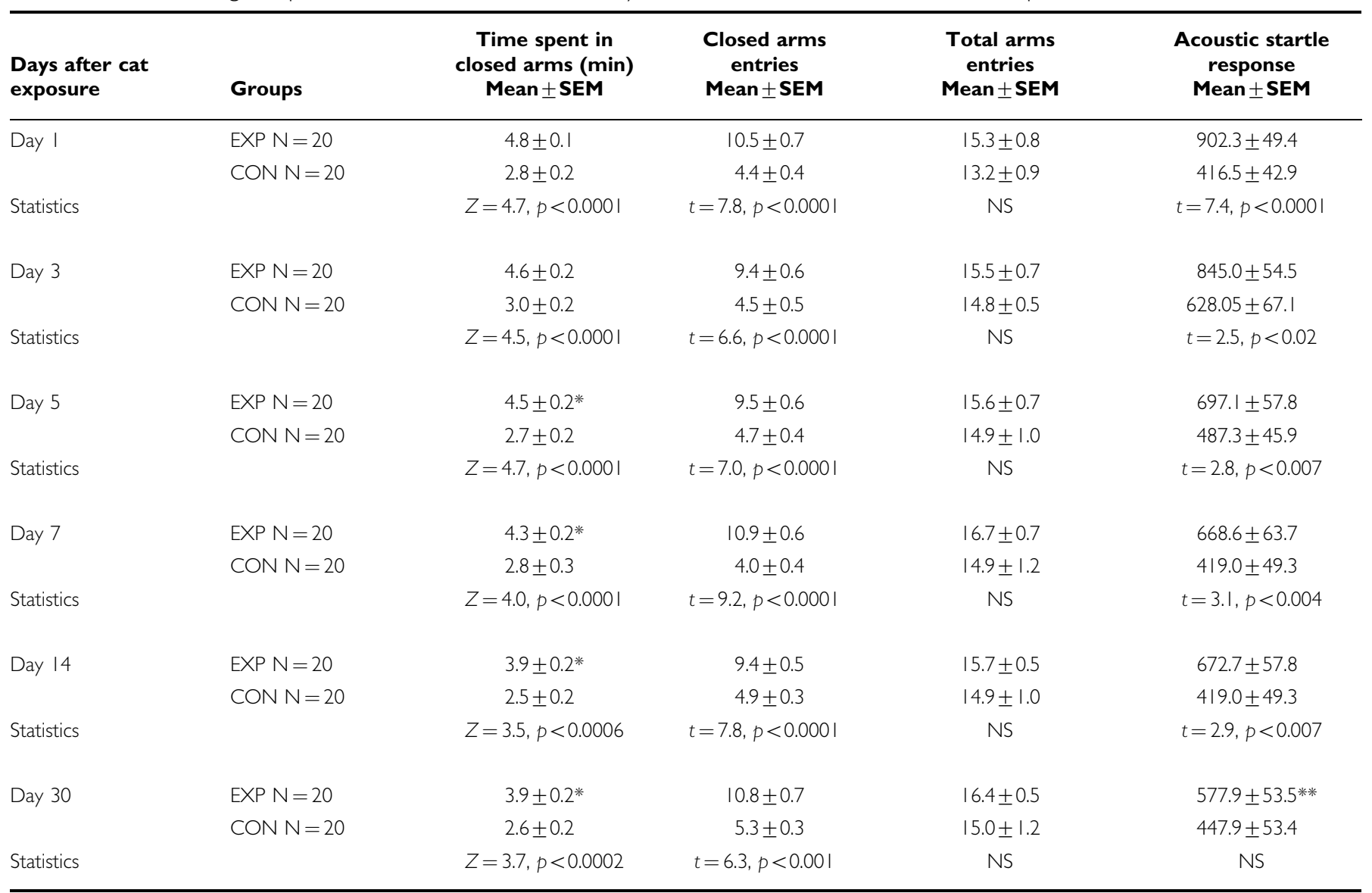

*Mann-Whitney U-test-significant difference vs exposed rats I day after exposure $(p<0.004)$.

**ANOVA — significant difference vs exposed rats I day after exposure $(p<0.008)$

criteria. However, Figure $2 \mathrm{~b}$ shows an increase in prevalence of MBR rats from 0 to $5 \%$ on days $1-7$ after cat exposure to $20 \%$ by day 30 .

All other rats fell between the $\mathrm{CBC}$ for the extreme groups, and were defined as subthreshold.

\section{Model II: The Underwater Trauma}

The effect of underwater trauma on anxiety-like behaviors. As shown in Table 2, underwater trauma caused significant avoidance of open spaces as compared to control (nonexposed) animals as measured 1-30 days after exposure. Underwater trauma-exposed rats spent significantly more time exploring the closed arms of the EPM $(p<0.001)$ and entered more frequently into the closed arms $(p<0.02)$ than the control rats. The total entries into the open and closed arms (total arms entries) of the plus maze were not reduced in all the experiments. (Thus, although the overall entry-score was unaffected, the animals blatantly preferred the closed to the open arms.)

When the data for the entire exposed population are analyzed dichotomously, there is no significant difference between the data for measures from successive sampling times.
The effect of underwater trauma on ASR. As shown in Table 2, underwater trauma significantly enhanced mean startle response as compared to control (nonexposure) as measured 1-30 days after the exposures $(p<0.002)$.

As above, the entire group shows no specific pattern of effect at successive sampling points over time.

Correlation of the time spent in the closed arms parameter and $A S R$. In the underwater trauma exposed group, there was a significant correlation between time spent in the closed arms and startle response $(r=0.7, \mathrm{~F}(1,34)=35.4$, $p<0.00001)$.

In the control group, there were no significant correlations between time spent in the closed arms and startle response.

\section{Reanalysis applying the $C B C$.}

The prevalence of maladapted rats: Figure $2 \mathrm{a}$ shows a steady drop in prevalence of maladapted rats from $91.6 \%$ on day 1 to $41.6 \%$ by day 7 . Prevalence rates at day 30 are not significantly different from those on day 7.

As shown in Figure 2b, minimal behavioral response animals were almost impossible to find among the exposed rats, during the first days after exposure, using two consecutive criteria. Figure $2 \mathrm{~b}$ shows an increase in 
a

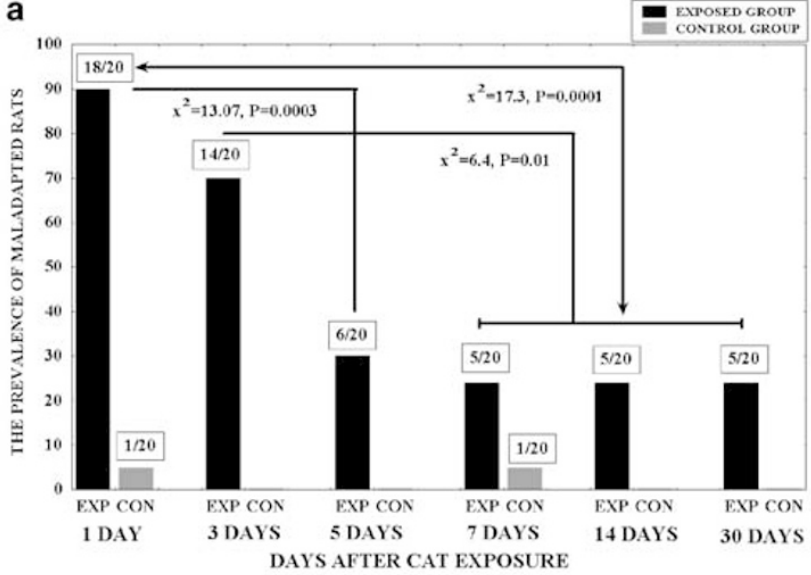

b

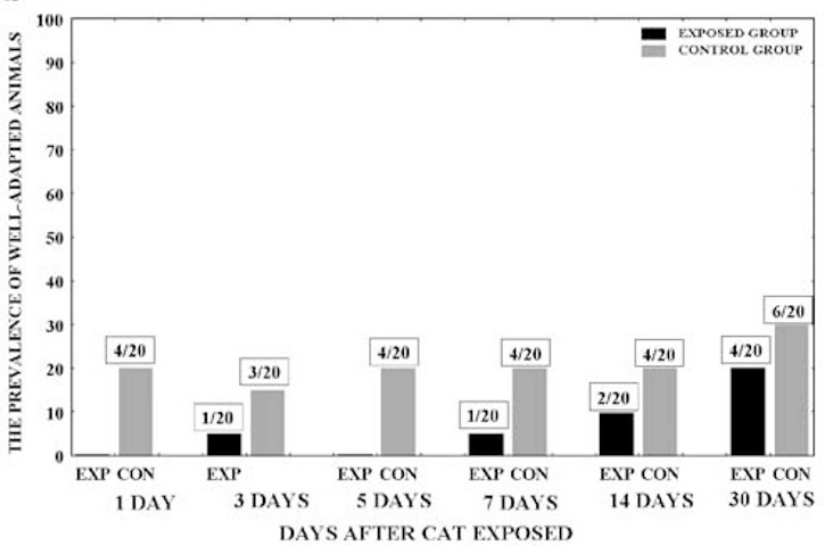

Figure I (a) Prevalence of maladapted rats after single exposure to predator. The figure shows a steady drop in prevalence of maladapted rats from $90 \%$ on day I to $25 \%$ by day 7 . As of day 7 , this rate remains constant until day 30. Our results have shown a progressive, time-dependent decline in the prevalence of MA rats (PTSD-like) during the first few weeks that follow a trauma. (b) Prevalence of well-adapted rats after single exposure to predator. The figure shows an increase in prevalence of well-adapted rats from 0 to $5 \%$ on days $1-7$ after cat exposure to $20 \%$ by day 30 . a

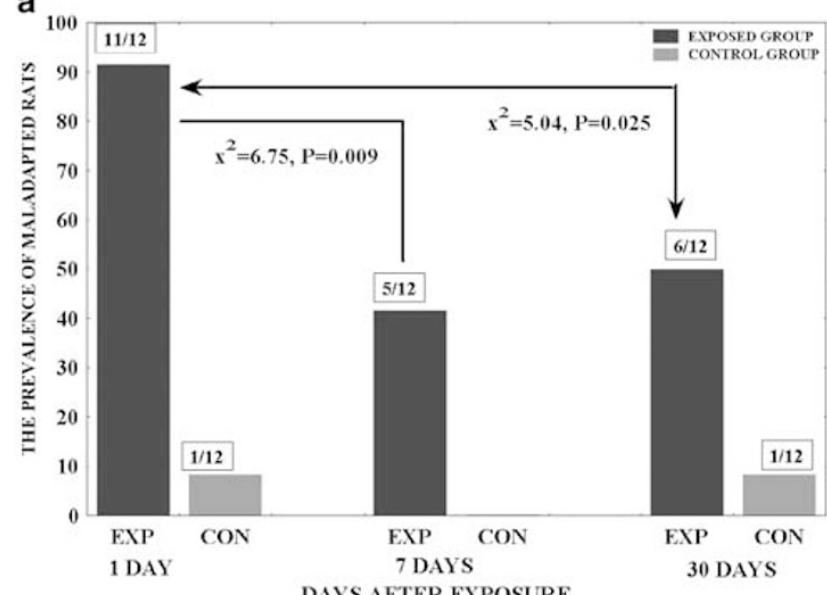

b

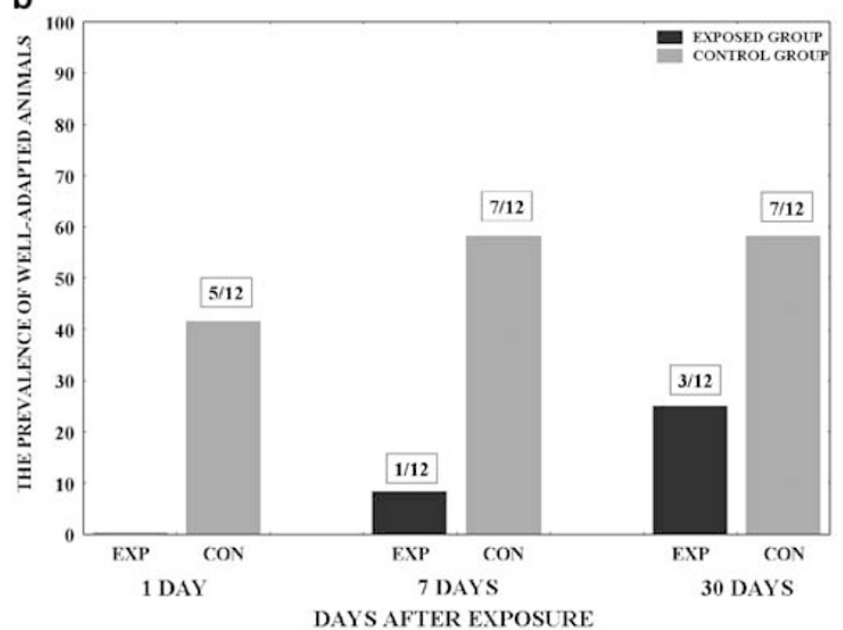

Figure 2 (a) Prevalence of maladapted rats after single underwater trauma exposure. The figure shows a steady drop in prevalence of maladapted rats from $91.6 \%$ on day I to $41.6 \%$ by day 7 . Prevalence rates at day 30 are not significantly different from those on day 7. (b) Prevalence of well-adapted rats after single underwater trauma exposure. The figure shows an increase in prevalence of well-adapted rats from $0 \%$ on day I after cat exposure to $25 \%$ by day 30 . All other rats fell between the CBC's for the extreme groups, and were defined as subthreshold.

Table 2 Effect of Underwater Trauma vs Control on Rat Anxiety-Like Behavior and Acoustic Startle Response

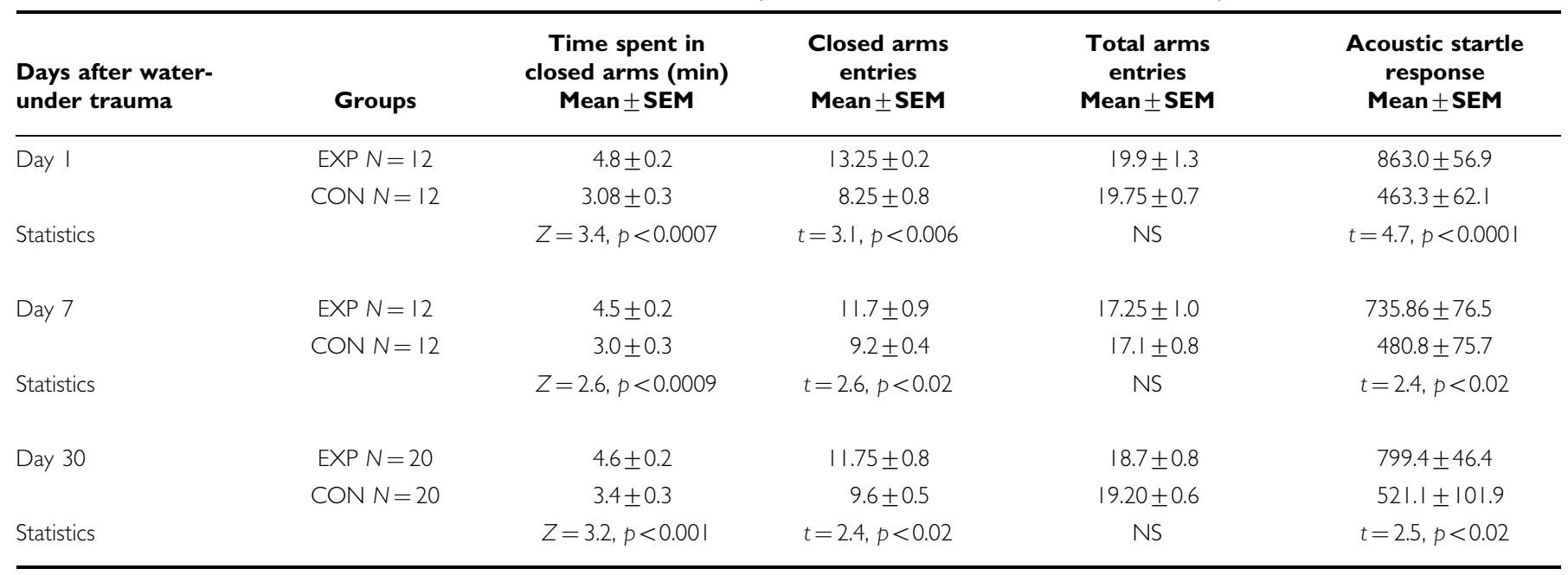


prevalence of MBR rats from $8.3 \%$ on day 7 after cat exposure to $25 \%$ by day 30 .

All other rats fell between the $\mathrm{CBC}$ for the extreme groups, and were defined as subthreshold.

\section{DISCUSSION}

In order for an animal behavioral model to serve its purpose and help to elucidate certain aspects of complex human clinical conditions comprising emotional, cognitive, existential, behavioral and physiological components, such as PTSD, the design of the model must strive to parallel the clinical condition as closely as possible.

Our model seeks to test the hypothesis that by means of clearly defining the animals most severely affected by a stressful experience, we will be able to focus on subpopulations of animals that have responded with a clearly defined degree of behavioral change over any specified period of time, whether these be the animals most severely affected, those least affected or those in-between. In this study, we focused on the most severely affected individuals over various time periods and in two distinct stress paradigms. In terms of extent and chronicity, these are the animals whose condition we think more closely resembles the symptoms characteristic of ASD and PTSD patients, rather than assuming that all exposed animals were equally affected.

Before testing our method of looking at the data from behavioral studies taking into consideration individual differences in degree of response, in each individual experiment, we always take care to establish that the data from the paradigm replicate those elicited in prior studies. The analysis of the above results where the exposed population was regarded as a homogeneous whole unequivocally demonstrated that, as a group, animals exposed to the stressors displayed a significant degree of anxiety-like behavioral changes over a significant period of time in the EPM and in the ASR paradigms. The accepted approach to analyzing anxious and fearful behavioral data from stress paradigms was thus borne out in each of the experiments.

The study proceeded to attempt to revise the procedure for defining an animal as having been affected by the stressful experience, by adding a second, consecutively performed behavioral paradigm for assessing a fearful/ anxious response as a second $\mathrm{CBC}$. The plus maze and ASR tests were selected to constitute the basis for the more demanding serial behavioral cutoff criteria for a number of reasons. Firstly, each has been shown to be a valid measure of behavioral stress responses in numerous studies. Secondly, they are each well defined and straightforward to score.

By virtue of separating out the more clearly affected animals, it became possible to define the prevalence of affected and unaffected animals at various time points, starting immediately after exposure to the stressor. Whereas analysis of the data en bloc shows that there is an overall response to the stressor, the differentiation according to $\mathrm{CBC}$ enables a curve to be plotted for each extreme of the behavioural response types. The prevalence of affected (maladapted) rats from among the cohort was shown to be very high (90\%) on day 1 and fairly rapidly dropped off to a constant prevalence rate of about $25 \%$ starting from day 7 through to day 30. Concomitantly, the prevalence rates of unaffected (WA) rats from among the exposed rats rose gradually from virtually none during the first days to a small although steadily increasing number per cohort as of day 5 . This pattern indicates that whereas immediately after the stressful and threatening experience a large proportion of the exposed population is quite clearly significantly affected by it, over the space of a fairly brief period of time many individuals show significantly less distress, and a gradually increasing proportion show no significant distress at all. In a sense, this trend resembles the pattern of response of populations followed up after exposure to traumatic events, that is, a large proportion of the exposed population may be expected to display quite significant distress at first, although many will show a steady tendency towards improvement in symptoms over the near future. A proportion may develop ASD and over time a minority, albeit a significant minority, will be found to fulfill criteria for the full-blown clinical syndrome of PTSD. The curve representing the prevalence rates of animal responses thus resembles the overall trends seen in human responses to the exposure to a significant stressor, and the proportions are numerically not unlike the gradual shift in symptom severity seen in populations over time (Yehuda et al, 1998). In this study, the pattern of an acute phase of stressinduced anxious/fearful responses demonstrated in most subjects immediately after exposure, followed by a gradual decline to chronic enduring effects found in a fairly constant proportion of subjects after several days or weeks, has been repeated in two distinctly different stress models-exposure to the scent of a predator on the one hand and underwater trauma on the other.

The results of these studies have also demonstrated a relationship between the type or character of the triggering stressor/trauma and the magnitude of the response among the population of exposed individuals, that is, the proportion of significantly affected individuals when analyzed according to the CBC. Underwater trauma, ie neardrowning, clearly elicits a more severe response than exposure to the scent of a predator's urine in a larger proportion of individual animals. In the same vein, there is a range of responses to various predator-exposure techniques. Actual exposure to a cat is more traumatic than being near a cat but protected by a dividing fence, and elicits higher proportions of significantly disrupted behavior, both of which are more stressful than exposure to predator-scent by being placed on soiled cat litter or a cat's blanket (unpublished data).

The fact that it is possible to distinguish between affected and unaffected individuals reaffirms the fact that animals display a range of responses to stimuli, as do the humans they are intended to model. The inclusion of all exposed animals in data analysis overlooks the individual variability in their behavioral response to the stressor, and represents a source for potential bias. Drawing a distinction between animals whom we choose to regard as affected to a sufficient degree by a stimulus in order to be included in a study from those whom we do not consider to have been sufficiently affected, may be seen as being conceptually similar to the application of inclusion and exclusion criteria when diagnosing patients or deciding whether to include them 
in a clinical study. This approach exposes patterns of responses which are significantly different from those seen when it is not applied, and which resemble patterns seen in human studies regarding the more severe type of long-term sequelae of exposure to stressors, including PTSD.

Whether PTSD is a disorder that reflects the upper end of a continuum of reactions to stress, as proposed by Ruscio et al (2002), or whether it is a distinct entity intimately related to, but not on a direct continuum with normal responses to stress (Yehuda and McFarlane, 1995), is at present a focus of discussion in the literature. The ability to distinguish and focus specifically on those who are affected and those who are not in animal models might help to shed light on questions such as this.

At any rate, the presented data suggest that it is important to perform a separate analysis for MA- and WA-exposed rats, as this approach not only takes into consideration, but intentionally highlights a range of individual differences in response to stress, whether they be qualitative or quantitative.

\section{LIMITATIONS}

One must take care not to be too literal in interpreting animal models. They are not to be taken to accurately reflect the human disorder, but merely to approximate certain aspects of it. It would be presumptuous to assume that the criteria applied in this study, in fact reflect psychophysiological parameters in the life of the rat, which are commensurate with the criteria for PTSD in human. The authors are not proposing that the criteria described or the behavioral paradigms used are the only appropriate ones for defining CBC. It is the rationale that has been tested.

Although the animals grow up together in near-identical environmental conditions in a vivarium, they are genetically distinct. A purebred strain study is under way to accommodate for this limitation, although we hypothesize that we shall nevertheless find a significant degree of individual variability.

The discarding of the data for the partial-responder rats should be taken as a temporary measure, aimed at a study of the clearcut data at the extremes, initially. In the future, this group may be the focus for the study of the spectrum of sequelae of stress.

\section{CONCLUSIONS}

This study is a preliminary body of work, which set out to further examine an alterative principle to the accepted dichotomous (exposed vs nonexposed) approach in animal models of PTSD. In this study, a method employing two consecutive cutoff (inclusion/exclusion) behavioral criteria (extreme fearfulness on the EPM and exaggerated ASR) was examined in two different animal models of potentially lifethreatening stress. We submit that classifying the exposed population into those who are behaviorally affected and those who are not affected clarifies the picture greatly, enabling patterns to emerge which had previously been obscured in animal studies where the method of data analysis had presumed the population of exposed animals to be homogenous, and had thus included data for unaffected individuals along with data for the affected.
The CBC model takes into account the individual variability in degree of response to the stress paradigm, that is, it shifts the focus from exposed vs nonexposed, to pathological vs virtually no response to the triggering stressful experience. It regards the heterogeneity in the behavioral response of the tested population as the key factor, the focal finding rather than a statistical epiphenomenon.

As the results from this study demonstrate quite convincing face validity, we propose that the potential effect on the predictive validity of treatment paradigms of applying this approach to analyzing data from animal models could be of great value. It is conceivable that the flexibility in emphasis onto the study of severely affected, partially affected, and unaffected populations in animal models might improve the study of treatment modalities for this incapacitating group of disorders.

Setting the affected individuals apart from the unaffected and focusing on each of them more closely, approximates the thinking behind clinical studies and might help to clarify some of the pending issues in stress and anxiety research.

\section{ACKNOWLEDGEMENTS}

This study was supported by Grant 52/2000 from the Israel Foundation Trusties to $G R-L$ and in part by a grant from NARSAD foundation to HC.

\section{REFERENCES}

Adamec R (1997). Transmitter systems involved in neural plasticity underlying increased anxiety and defense-implications for understanding anxiety following traumatic stress. Neurosci Biobehav Rev 21: 755-765.

Adamec R, Kent P, Anisman H, Shallow T, Merali Z (1998). Neural plasticity, neuropeptides and anxiety in animals - implications for understanding and treating affective disorder following traumatic stress in humans. Neurosci Biobehav Rev 23: 301-318.

Adamec RE, Burton P, Shallow T, Budgell J (1999). NMDA receptors mediate lasting increases in anxiety-like behavior produced by the stress of predator exposure - implications for anxiety associated with posttraumatic stress disorder. Physiol Behav 65: 723-737.

Adamec RE, Shallow T (1993). Lasting effects on rodent anxiety of a single exposure to a cat. Physiol Behav 54: 101-109.

Adamec RE, Shallow T, Budgell J (1997). Blockade of CCK(B) but not $\operatorname{CCK}(\mathrm{A})$ receptors before and after the stress of predator exposure prevents lasting increases in anxiety-like behavior: implications for anxiety associated with posttraumatic stress disorder. Behav Neurosci 111: 435-449.

American Psychiatric Association (1994). Diagnostic and Statistical Manual of Mental Disorders, 4th edn. American Psychiatric Association: Washington, DC.

Blanchard RJ, Blanchard DC, Rodgers J, Weiss SM (1990). The characterization and modelling of antipredator defensive behavior. Neurosci Biobehav Rev 14: 463-472.

Blanchard RJ, Blanchard DC, Weiss SM, Meyer S (1990b). The effects of ethanol and diazepam on reactions to predatory odors. Pharmacol Biochem Behav 35: 775-780.

Blanchard RJ, Nikulina JN, Sakai RR, McKittrick C, McEwen B, Blanchard DC (1998). Behavioral and endocrine change following chronic predatory stress. Physiol Behav 63: 561-569.

Blanchard RJ, Shepherd JK, Rodgers RJ, Magee L, Blanchard DC (1993). Attenuation of antipredator defensive behavior in rats 
following chronic treatment with imipramine. Psychopharmacology 110: 245-253.

Breslau N, Davis GC, Andreski P, Peterson E (1991). Traumatic events and posttraumatic stress disorder in an urban population of young adults. Arch Gen Psychiatry 48: 216-222.

Breslau N, Kessler RC, Chilcoat HD, Schultz LR, Davis GC, Andreski P (1998). Trauma and posttraumatic stress disorder in the community: the 1996 Detroit Area Survey of Trauma. Arch Gen Psychiatry 55: 626-632.

Cohen H, Benjamin J, Kaplan Z, Kotler M (2000). Administration of high-dose ketoconazole, an inhibitor of steroid synthesis, prevents posttraumatic anxiety in an animal model. Eur Neuropsychopharmacol 10: 429-435.

Cohen H, Friedberg S, Michael M, Kotler M, Zeev K (1996). Interaction of CCK-4 induced anxiety and post-cat exposure anxiety in rats. Depress Anxiety 4: 144-145.

Cohen H, Joseph Z, Matar M (2003). The relevance of differential response to trauma in an animal model of post-traumatic stress disorder. Biol Psychiatry 15: 463-473.

Cohen H, Kaplan Z, Kotler M (1999). CCK-antagonists in a rat exposed to acute stress: implication for anxiety associated with post-traumatic stress disorder. Depress Anxiety 10: 8-17.

Fairbank JA, Ebert L, Costello EJ (2000). Epidemiology of traumatic events and post-traumatic stress disorder. In: Nutt D, Davidson JRT, Zohar J (eds). Post Traumatic Stress Disorder: Diagnosis, Management and Treatment. Martin Dunitz Ltd: London. pp 17-27.

File SE (1993). The interplay of learning and anxiety in the elevated plus-maze. Behav Brain Res 58: 199-202.

Griebel G, Blanchard DC, Agnes RS, Blanchard RJ (1995a). Differential modulation of antipredator defensive behavior in Swiss-Webster mice following acute or chronic administration of imipramine and fluoxetine. Psychopharmacology (Berl) 120: 57-66.

Griebel G, Blanchard DC, Jung A, Lee JC, Masuda CK, Blanchard RJ (1995b). Further evidence that the mouse defense test battery is useful for screening anxiolytic and panicolytic drugs: effects of acute and chronic treatment with alprazolam. Neuropharmacology 34: 1625-1633.

Helzer JE, Robins LN, McEvoy L (1987). Post-traumatic stress disorder in the general population. Findings of the epidemiologic catchment area survey. $N$ Engl J Med 317: 1630-1634.

Kessler RC, Sonnega A, Bromet E, Hughes M, Nelson CB (1995). Posttraumatic stress disorder in the National Comorbidity Survey. Arch Gen Psychiatry 52: 1048-1060.
Perkonigg A, Kessler RC, Storz S, Wittchen HU (2000). Traumatic events and post-traumatic stress disorder in the community: prevalence, risk factors and comorbidity. Acta Psychiatr Scand 101: 46-59.

Pynoos RS, Ritzmann RF, Steinberg AM, Goenjian A, Prisecaru I (1996). A behavioral animal model of posttraumatic stress disorder featuring repeated exposure to situational reminders. Biol Psychiatry 39: 129-134.

Resnick HS, Kilpatrick DG, Dansky BS, Saunders BE, Best CL (1993). Prevalence of civilian trauma and posttraumatic stress disorder in a representative national sample of women. J Consult Clin Psychol 61: 984-991.

Richter-Levin G (1998). Acute and long-term behavioral correlates of underwater trauma-potential relevance to stress and poststress syndromes. Psychiatry Res 79: 73-83.

Rodgers RJ, Blanchard DC, Wong LK, Blanchard RJ (1990). Effects of scopolamine on antipredator defense reactions in wild and laboratory rats. Pharmacol Biochem Behav 36: 575-583.

Ruscio AM, Ruscio J, Keane TM (2002). The latent structure of posttraumatic stress disorder: a taxometric investigation of reactions to extreme stress. J Abnorm Psychol 111: 290-301.

Servatius RJ, Ottenweller JE, Natelson BH (1995). Delayed startle sensitization distinguishes rats exposed to one or three stress sessions: further evidence toward an animal model of PTSD. Biol Psychiatry 38: 539-546.

Shalev AY (2000). Post-traumatic stress disorder: diagnosis, history and life course. In: Nutt D, Davidson JRT, Zohar J (eds). Post-traumatic Stress Disorder: Diagnosis, Management and Treatment. Martin Dunitz Ltd: London. pp 1-15.

Shepherd JK, Flores T, Rodgers RJ, Blanchard RJ, Blanchard DC (1992). The anxiety/defense test battery: influence of gender and ritanserin treatment on antipredator defensive behavior. Physiol Behav 51: 277-285.

Shore JH, Vollmer WM, Tatum EL (1989). Community patterns of posttraumatic stress disorders. J Nerv Ment Dis 177: 681-685.

Stam R, Bruijnzeel AW, Wiegant VM (2000). Long-lasting stress sensitisation. Eur J Pharmacol 405: 217-224.

Wang J, Akirav I, Richter-Levin G (2000). Short-term behavioral and electrophysiological consequences of underwater trauma. Physiol Behav 70: 327-332.

Yehuda R, McFarlane AC (1995). Conflict between current knowledge about posttraumatic stress disorder and its original conceptual basis. Am J Psychiatry 152: 1705-1713.

Yehuda R, McFarlane AC, Shalev AY (1998). Predicting the development of posttraumatic stress disorder from the acute response to a traumatic event. Biol Psychiatry 44: 1305-1313. 